\title{
Identification of a Novel Ryanodine Receptor Mutation Causing Malignant Hyperthermia
}

\author{
Iveta Valaskova, ${ }^{1,2}$ Silvie Dudova, ${ }^{1,2}$ Jana Necasova, ${ }^{1}$ \\ Edita Ostadalova, ${ }^{1}$ Martina Vanaskova, ${ }^{1}$ Dagmar Stepankova, ${ }^{3}$ \\ Ivana Schröderova, ${ }^{4}$ Renata Gaillyova, ${ }^{1,2}$ and Petr Kuglik ${ }^{1,5}$ \\ ${ }^{1}$ Department of Medical Genetics, University Hospital Brno, Cernopolni 9, 61300 Brno, Czech Republic \\ ${ }^{2}$ Department of Biology, Faculty of Medicine, Masaryk University, Kamenice 5, 62500 Brno, Czech Republic \\ ${ }^{3}$ Clinic of Paediatric Anaesthesiology and Intensive Care, University Hospital Brno, Cernopolni 9, 61300 Brno, Czech Republic \\ ${ }^{4}$ Department of Anaesthesiology and Intensive Care, St. Anne's Faculty Hospital, Pekarska 53, 65651 Brno, Czech Republic \\ ${ }^{5}$ Department of Experimental Biology, Masaryk University, Kotlarska 267/2, 61137 Brno, Czech Republic
}

Correspondence should be addressed to Iveta Valaskova; ivalaskova@fnbrno.cz

Received 27 August 2013; Accepted 1 October 2013

Academic Editors: A. Brehm, A. Wang, and A. Yamamoto

Copyright (c) 2013 Iveta Valaskova et al. This is an open access article distributed under the Creative Commons Attribution License, which permits unrestricted use, distribution, and reproduction in any medium, provided the original work is properly cited.

\begin{abstract}
Malignant hyperthermia $(\mathrm{MH})$ is a potentially fatal autosomal dominant disorder of the skeletal muscle and is triggered in susceptible individuals by commonly used inhalation anaesthetics and depolarizing muscle relaxants. Around $80 \%$ of the affected family are linked to the ryanodine receptor (RYR1) gene. More than 300 mutations in RYR1 have been associated with the MHsusceptible phenotype. Here we report the identification by two independent methods of a novel mutation associated with the $\mathrm{MH}$-susceptible phenotype in the RYR1 gene.
\end{abstract}

\section{Introduction}

Malignant hyperthermia susceptibility (MHS; OMIM * 145600) is an autosomal, dominantly inherited, potentially fatal pharmacogenetic disturbance of intracellular calcium homeostasis, associated with the ryanodine receptor 1 (RYRl; OMIM * 180901) in the sarcoplasmic reticulum of the skeletal muscle cells. In susceptible individuals, volatile or inhalational anaesthetics such as halothane and depolarizing muscle relaxants like succinylcholine can induce severe decompensation of muscle calcium homeostasis [1].

Clinical symptoms of an $\mathrm{MH}$ attack are hyperthermia, accelerated muscle metabolism, muscle contractions, metabolic acidosis, and tachycardia, and unless immediately recognised, and treated it is fatal [1].

Because $\mathrm{MH}$ is a subclinical myopathy, MHS individuals are asymptomatic during daily life. Outside anaesthesia, the diagnosis of MHS can only be established with specific testing by an in vitro contracture test (IVCT), based on the differential contractile response of normal (MHN) and MHS muscles to halothane and caffeine [2]. IVCT is expensive and invasive as it requires an open muscle biopsy from the quadriceps femoris. Genetic screening of the ryanodine receptor isoform 1 (RYR1) is an alternative method for identifying probands and their family members as $\mathrm{MH}$-susceptible after clinical evidence of MH susceptibility [3]. Molecular genetic testing indicates that mutations in $R Y R 1$ are identified in up to $70 \%-$ $80 \%$ of the individuals with confirmed MHS [4]. The majority of mutations appear to be clustered in three hotspots: between exons 2 and 17 encoding amino acid residues from 35 to 614 (MH region 1), between exons 39 and 46 encoding amino acid residues from 2163 to 2458 ( $\mathrm{MH}$ region 2), and a third region in the $3^{\prime}$ end of the gene is between exons 90 and 106 encoding amino acid residues from 4668 to 4904 (MH region 3) [5].

Here, we report a novel mutation located in the central portion of the RYR1 gene in a Czech family.

\section{Materials and Methods}

We investigated a multigenerational Czech family with two known fatal MH reactions. Four members of the family 


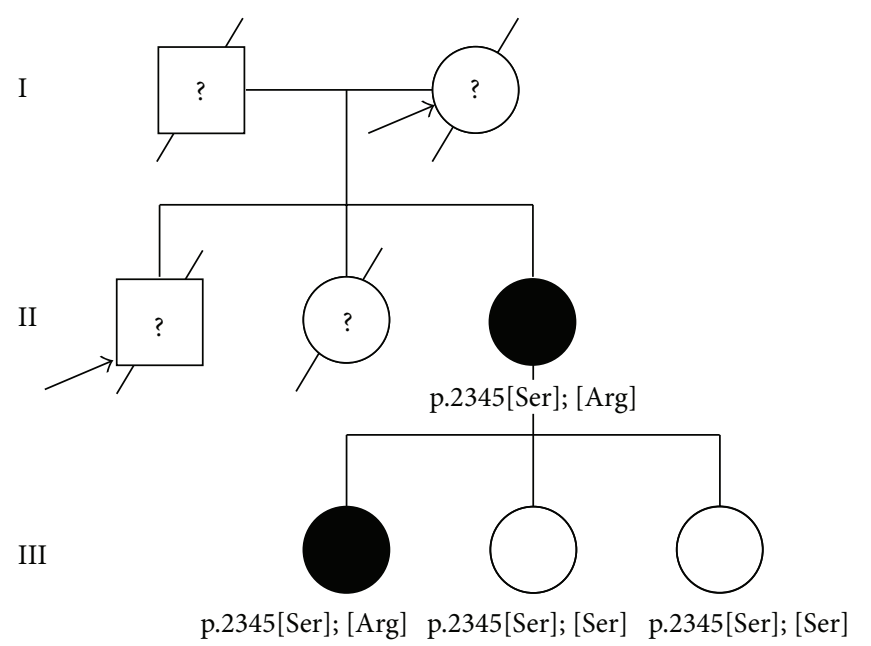

FIGURE 1: Segregation data in a family carrying novel RYR1 variant p.Ser2345Arg. Solid symbols denote MHS status identified by IVCT, open symbols denote IVCT-typed MHN individuals, and question marks indicate that IVCT had not been performed. The arrows indicate index cases that experienced an MH episode.

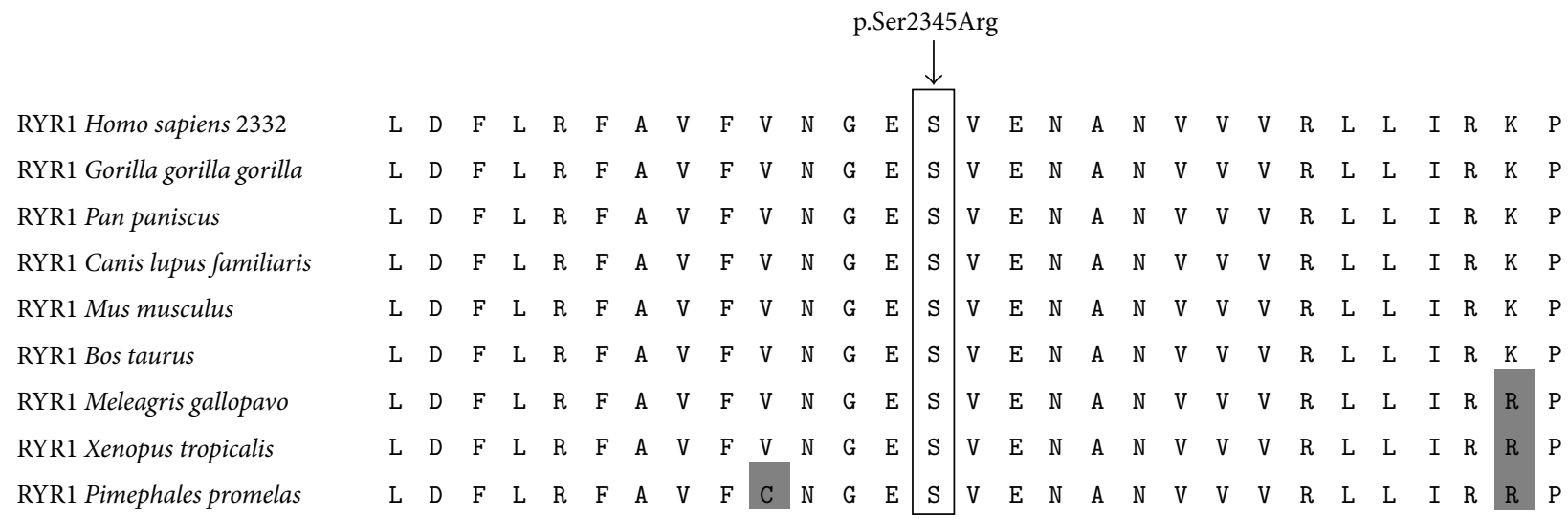

FIgURE 2: Amino acid conservation of the RYR1 region where the novel sequence variant was detected. For the p.Ser2345Arg mutation, the relevant cutout from the amino acid sequence of RYR1 is aligned to the RYR sequences from other species. Amino acids are denoted by the single-letter code. Amino acids divergent from human RYR1 are shaded. The position of p.Ser2345Arg is indicated by an arrow. To illustrate the evolutionary conservation of the RYR1 region where the novel sequence variant occurred, the corresponding sequences from other vertebrates are given: gorilla (Gorilla gorilla gorilla), chimpanzee (Pan paniscus), dog (Canis lupus familiaris), mouse (Mus musculus), cow (Bos taurus) from mammals, turkey (Meleagris gallopavo) from birds, frog (Xenopus tropicalis) from amphibian, and minnow (Pimephales promelas) from fish.

underwent the IVCT. The novel variant was detected by direct sequencing of RYR1-cDNA hotspots. Total RNA was extracted from muscle tissue biopsied at the time of the IVCTs using Trizol (Invitrogen), and cDNA was synthesized using Sensiscript RT (Qiagen). cDNA strands were amplified in 600-650 bp overlapping fragments covering most reported RYR1 hotspots. PCR reactions were carried out using the Hot Start Master Mix Kit (Qiagen). The primer sequences and PCR conditions are available from the authors on request. PCR products were bidirectionally sequenced using the BIG DYE dideoxy-terminator chemistry (Applied Biosystems) on an ABI 3130 (Applied Biosystems) automatic sequencer. The novel variant was confirmed by direct sequencing of the relevant exon of the RYR1. The genomic DNA was extracted from peripheral blood.
Two hundred DNA samples from clinically healthy subjects were investigated for the absence of the unknown sequence change to exclude population specific polymorphism. The MH status of 150 subjects was not known. Fifty controls were classified as MH-negative by IVCT performed for suspected $\mathrm{MH}$.

\section{Results}

In a multigenerational family with a severe form of malignant hyperthermia that has caused two deaths, a novel missense heterozygous substitution of $\mathrm{C}>\mathrm{T}$ at position 7033 of c.DNA that changes a serine to arginine at position 2345 of the protein sequence (c.7033G > T; p.Ser2345Arg) was identified within the RYR1 gene. This sequence change has been tested 
by a segregation and conservation study to elucidate its significance as a causative mutation. Our segregation data were limited by the number of family members IVCT tested. However, the variant perfectly cosegregated with MHS status in the small nuclear family available (Figure 1). The variant p.S2345R was carried by two individuals testing positive for MH susceptibility by IVCT, but absent from two MHN individuals.

\section{Discussion}

This study has identified a novel inherited DNA variant within the RYR1 gene. This variant is a nucleotide substitution that results in a change of amino acid residue, which possibly associates with alteration of the ryanodine protein structure. Its central domain location might enable the structural modification and aberrant response of the ryanodine receptor, resulting in a leaky $\mathrm{Ca}^{2+}$ channel [6]. The novel mutation p.Ser2345Arg described here is located close to the previously identified $\mathrm{MH}$ associated mutations: p.Asn2342Ser [7], p.Glu2344Asp [8], p.Val2346Met [9], and p.Ala2350Thr [10].

The detected substitution affects an amino acid which is highly conserved through evolution (Figure 2). Conservation of protein domains over long evolutionary distances is generally thought to indicate functional relevance. Substitution of an amino acid within such a domain is, therefore, regarded as likely to compromise the function of the protein with the potential sequela of disease [2].

This variant was absent from 400 control RYR1 alleles. This observation indicates that the replacement of Ser2345 with an arginine residue is very unlikely to be polymorphism.

The p.Ser2345Arg mutation has been identified so far only in one family (out of $47 \mathrm{MHS}$ unrelated subjects) and strictly segregates with the MHS phenotype. The described mutation occurred in two MHS family members testing positive for MHS by IVCT. Moreover, no other RYR1 mutations, among $31 \mathrm{MH}$ causative mutations analysed, were detected in this pedigree.

On the basis of these arguments, the novel mutation described should be considered as a candidate mutation for MH. Therefore, relatives who have not yet been tested by IVCT should be regarded as MHS for clinical purposes, since they bear the p.Ser2345Arg substitution with high-risk familial haplotype. However, according to the guidelines for molecular genetic detection of susceptibility to malignant hyperthermia [11], we recommended to family members who do not carry the mutation observed in pedigree that they still undergo IVCT investigation.

\section{Conflict of Interests}

The authors declare that there is no conflict of interests.

\section{References}

[1] K. M. Stowell, "Malignant hyperthermia: a pharmacogenetic disorder," Pharmacogenomics, vol. 9, no. 11, pp. 1657-1672, 2008.
[2] H. Ørding, V. Brancadoro, S. Cozzolino et al., "In vitro contracture test for diagnosis of malignant hyperthermia following the protocol of the European $\mathrm{MH}$ group: results of testing patients surviving fulminant $\mathrm{MH}$ and unrelated low-risk subjects," Acta Anaesthesiologica Scandinavica, vol. 41, no. 8, pp. 955-966, 1997.

[3] A. Urwyler, T. Deufel, T. McCarthy, and S. West, "Guidelines for molecular genetic detection of susceptibility to malignant hyperthermia," British Journal of Anaesthesia, vol. 86, no. 2, pp. 283-287, 2001.

[4] N. Sambuughin, H. Holley, S. Muldoon et al., "Screening of the entire ryanodine receptor type 1 coding region for sequence variants associated with malignant hyperthermia susceptibility in the North American population," Anesthesiology, vol. 102, no. 3, pp. 515-521, 2005.

[5] R. Robinson, D. Carpenter, M.-A. Shaw, J. Halsall, and P. Hopkins, "Mutations in RYR1 in malignant hypertheraiia and central core disease," Human Mutation, vol. 27, no. 10, pp. 977-989, 2006.

[6] K. Jurkat-Rott, T. V. McCarthy, and F. Lehmann-Horn, "Genetics and pathogenesis of malignant hyperthermia," Muscle \& Nerve, vol. 23, pp. 4-17, 2000.

[7] C. L. Marchant, F. R. Ellis, P. J. Halsall, P. M. Hopkins, and R. L. Robinson, "Mutation analysis of two patients with hypokalemic periodic paralysis and suspected malignant hyperthermia," Muscle and Nerve, vol. 30, no. 1, pp. 114-117, 2004.

[8] N. Monnier, G. Kozak-Ribbens, R. Krivosic-Horber et al., "Correlations between genotype and pharmacological, histological, functional, and clinical phenotypes in malignant hyperthermia susceptibility," Human Mutation, vol. 26, no. 5, pp. 413-425, 2005.

[9] S. Shepherd, F. Ellis, J. Halsall, P. Hopkins, and R. Robinson, "RYR1 mutations in UK central core disease patients: more than just the C-terminal transmembrane region of the RYR1 gene," Journal of Medical Genetics, vol. 41, no. 3, article e33, 2004.

[10] K. A. Quane, J. M. S. Healy, K. E. Keating et al., "Mutations in the ryanodine receptor gene in central core disease and malignant hyperthermia," Nature Genetics, vol. 5, no. 1, pp. 51-55, 1993.

[11] M. Broman, A. Gehrig, G. Islander et al., "Mutation screening of the RYR1-cDNA from peripheral B-lymphocytes in 15 swedish malignant hyperthermia index cases," British Journal of Anaesthesia, vol. 102, no. 5, pp. 642-649, 2009. 

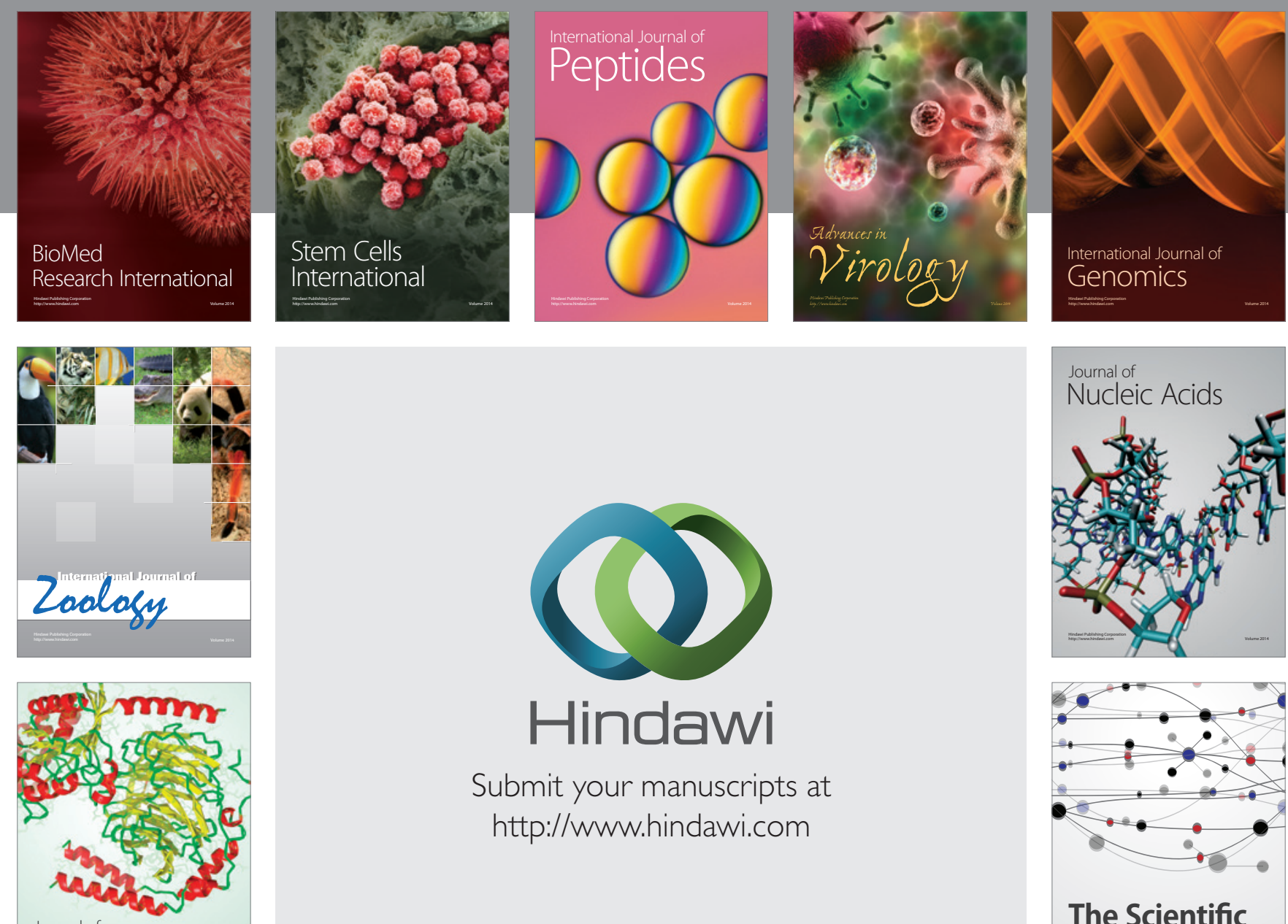

Submit your manuscripts at

http://www.hindawi.com

Journal of
Signal Transduction
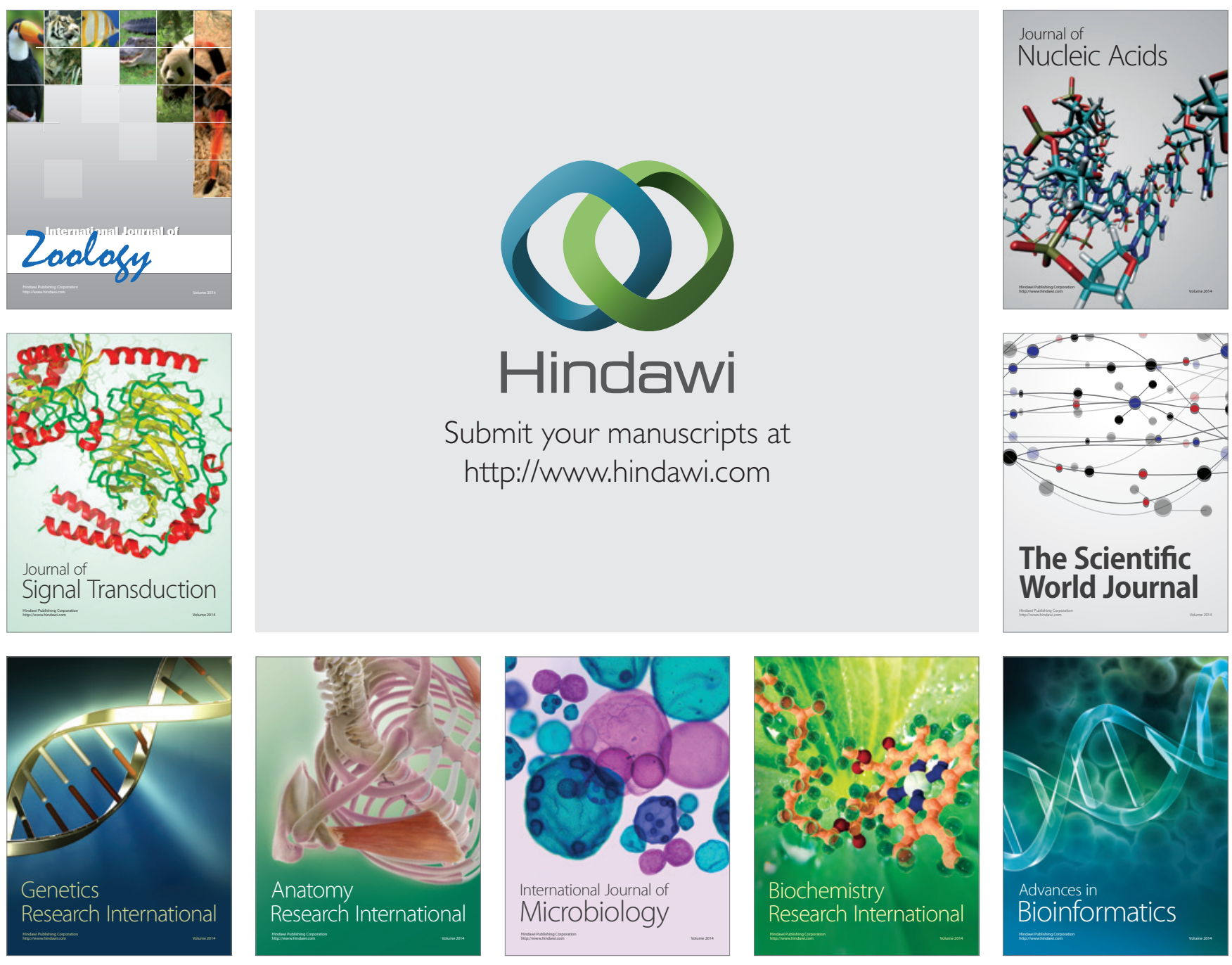

The Scientific World Journal
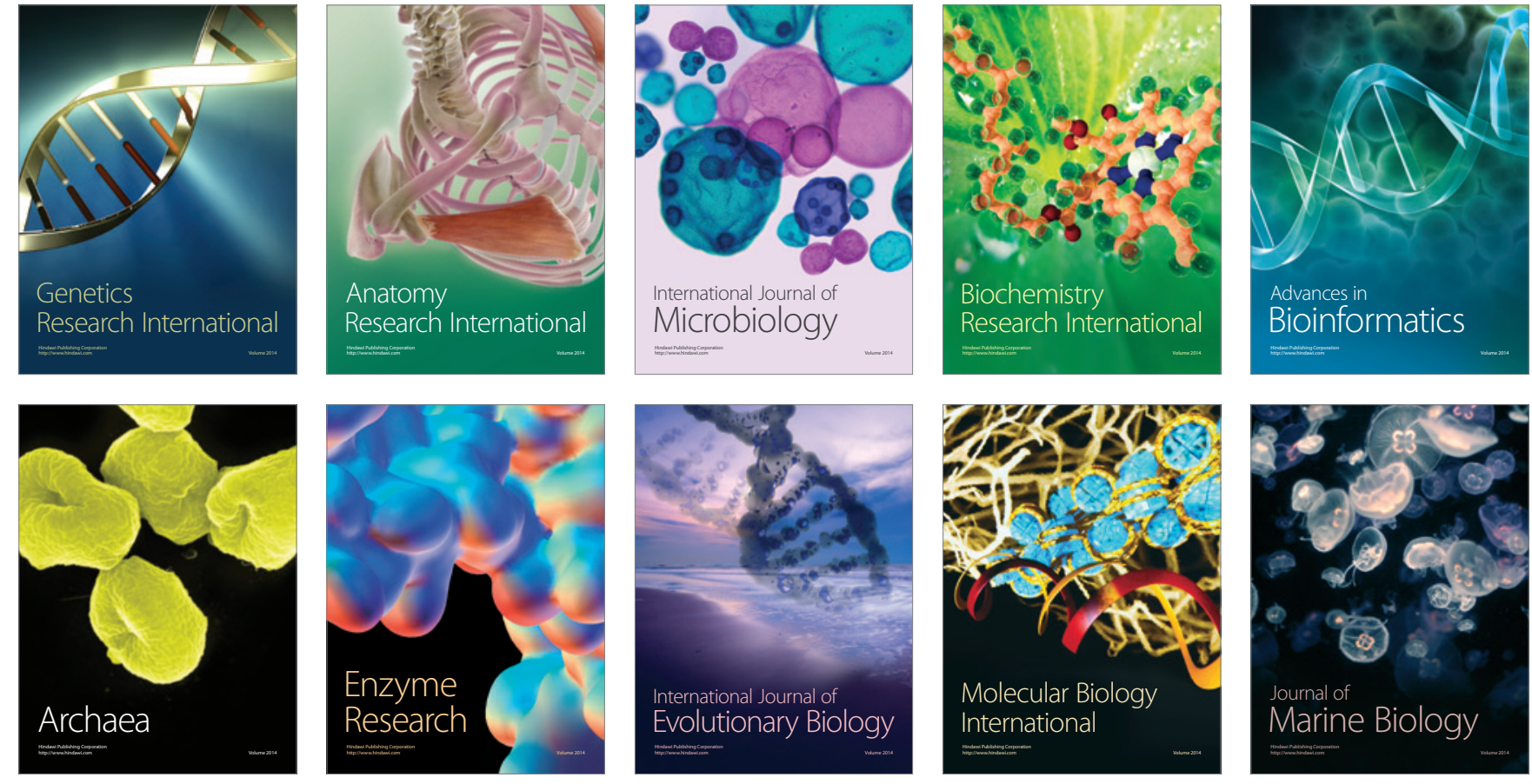